\title{
Un centre des archives du monde du travail pour les recherches en sciences de la communication
}

\section{Pierre Delcambre}

\section{(2) OpenEdition}

1 Journals

Édition électronique

URL : http://journals.openedition.org/communicationorganisation/3032

DOI : 10.4000/communicationorganisation.3032

ISSN : $1775-3546$

Éditeur

Presses universitaires de Bordeaux

Édition imprimée

Date de publication : 1 novembre 1996

ISSN : 1168-5549

Référence électronique

Pierre Delcambre, « Un centre des archives du monde du travail pour les recherches en sciences de la communication », Communication et organisation [En ligne], 10 | 1996, mis en ligne le 01 avril 2012 consulté le 01 mai 2019. URL : http://journals.openedition.org/communicationorganisation/3032 ; DOI : 10.4000/communicationorganisation.3032

Ce document a été généré automatiquement le 1 mai 2019.

(c) Presses universitaires de Bordeaux 


\title{
Un centre des archives du monde du travail pour les recherches en sciences de la communication
}

\author{
Pierre Delcambre
}

1 Quand Hugues Hotier m'a demandé de présenter le Centre des archives du monde du travail (CAMT) je me suis d'abord demandé : à quel titre vais-je parler? Je ne suis pas le chargé de relations publiques de cette institution. Certes, je suis le régional de l'étape, puisque le CAMT, est situé à Roubaix, à deux pas de l'IUP Infocom où j'enseigne.

2 Je ne suis pas non plus un expert du travail sur archives. Il m'est certes arrivé de travailler sur archives d'entreprises, dans les locaux d'institutions diverses; mais je ne suis pas un spécialiste du travail historique dans le domaine de la communication et de la communication organisationnelle.

En fait, je suis un utilisateur des archives d'entreprises, un Partenaire habituel du CAMT. Et c'est à ce titre que j'accepte de participer à sa promotion, dans ce texte. Il ne s'agira pas d'un texte purement promotionnel : il m'a semblé utile de concevoir cette intervention en commençant par une présentation des missions du CAMT et du sens que les dirigeants de cette institution donnent à leur activité. Pour ce faire, je reprendrai une interview du directeur du CAMT, réalisée en février 1995 pour Études de communication. Ensuite, j'indiquerai les questions et les intérêts de l'échange entre conservateurs et chercheurs en sciences de la communication. Je reprendrai une interview réalisée dans les mêmes conditions auprès d'A. Le Goff, conservatrice dans le même Centre. Enfin, je terminerai ce papier en présentant deux fonds nouvellement constitués à Roubaix et en signalant les différents documents de cette institution qui permettent aux chercheurs en sciences de l'information et de la communication de penser et de programmer un travail dans ces fonds d'une richesse peu soupçonnée. 


\section{Un centre de ressources pour comprendre les entreprises du temps présent...}

Les chercheurs des sciences de la communication ne sont pas, pour la plupart, des historiens. Ils travaillent le plus souvent sur des phénomènes contemporains, cherchent à comprendre des évolutions, des mutations. Que ce soit à propos de vie locale, d'entreprises, de médias...

5 Pourtant, pour ne pas être prisonniers d'une illusion de "modernité ", il leur faut souvent adopter le regard historique qui cherche les lignes de fracture, les répétitions, les situations où « plus ça change, plus c'est toujours la même chose...

6 Aussi y a-t-il lieu de faire connaître aux chercheurs un nouvel outil pour leurs travaux, ouvert au public depuis 1993, ayant accueilli les archives dites industrielles (séries AQ) de la Bibliothèque nationale depuis juillet 1996 : le Centre des archives du monde du travail (Roubaix).

\section{Entretien avec G. Mouradian, directeur du Centre des archives du monde du travail.}

\section{Qu'est-ce qu'un Centre des archives du inonde du travail ?}

Question: Le titre « archives du monde du mravail » est une nouvelle dénomination en France. Quel projet institutionnel recouvre un tel terme?

8 La notion française s'est constituée dans les années 1970, quand on s'est préoccupé vraiment de trouver d'autres sources que les Archives publiques - de façon importante en tout cas, puisque la collecte d'archives d'entreprises date de l'entre-deux guerres. Le mouvement de 68 a incité à ouvrir de nouveaux champs à la recherche et à trouver des sources issues directement des acteurs sociaux de l'entreprise : syndicats, associations, entreprises. S'y sont ajoutées la crise, la casse industrielle, qui ont abouti à la fermeture d'entreprises. On s'est alors préoccupé, dans l'urgence, de la mémoire des archives privées produites par l'entreprise. En France, on a développé le concept du «monde du travail ", officialisé comme terminologie dans les années 80. Cette notion est assez différente de celle qui est développée à l'étranger où il y a une césure assez forte entre les archives d'entreprise (Business Archives selon le terme anglo-saxon) et les Centres d'histoire sociale où associations, organismes du mouvement syndical et partis politiques se sont préoccupés de regrouper leurs documentations d'abord, leurs archives ensuite. En France, on a considéré que les Archives du monde du travail étaient celles des acteurs sociaux de la production, ceux qui interviennent dans le champ des rapports sociaux de production, de manière organisée ou à titre personnel. On pourra donc ici trouver aussi des archives personnelles de militants, des archives personnelles patronales.

Ainsi, en France, on a trouvé qu'il était plus pertinent de regrouper les deux versants du procès de production : archives d'entreprises économiques, archives syndicales. Question: Et, en conséquence, seront exclues les archives des partis politiques?

11 Tout à fait, c'est bien là la différence entre notre manière de faire et celle que l'on trouvera en Belgique, aux Pays-Bas, en Allemagne, en Italie... où les archives sont 
organisées selon une césure entre l'entreprise et le mouvement ouvrier au sens large, qu'il soit d'obédience chrétienne, sociale-démocrate ou marxiste. En France, ce sont les Archives nationales et départementales, mais aussi des institutions autour de l'Université, l'Institut historique du temps présent notamment, qui collectent les archives politiques... et cherchent d'ailleurs à collecter aussi les archives syndicales, sur le mode de leurs collègues étrangers.

\section{Les documents collectés. Écomusées et Archives du monde du travail.}

Question: Quelle est la nature des supports que vous collectez?

13 Les archives, au sens de l'article premier de la loi de 79, n'excluent pas un support ou un autre, tant que les documents portent de l'information et sont produits dans le cadre d'une action. A priori on n'a pas vocation - mais certes il y a des problèmes frontaliers - à être un centre de mémoire pour une ethnographie du monde du travail, par exemple. On trouvera essentiellement le support papier, la vidéo, la photo, mais encore parfois des documents qui se rapprochent de l'objet - d'où les problèmes frontaliers que j'évoquais registres d'échantillons de filatures, logos, étiquettes; documents techniques; il s'agit là de documents qui sont intimement liés à la production de l'entreprise et sont donc archives de l'entreprise... Et en même temps les musées des Arts et des Techniques, les musées d'Arts appliqués s'intéresseront aussi à la collecte de ces documents. Autant alors ne pas se situer en termes de rivalité et faire comprendre ce que notre collecte a de spécifique : une institution muséographique, quand elle procède à la collecte de ce genre d'objet va avoir tendance à détruire la partie archives papier, écrits, pour ne collecter que cela. Alors que nous, autant s'accorder de temps en temps un peu de mérite, on a effectivement une action cohérente sur cet ensemble de documentation. Un document vaut dans son contexte de production.

14 Il faut ajouter un point: nous nous préoccupons de collecte pérenne. Les Archives du monde du travail ont été créées au cœur de la crise de la société industrielle, on pourrait dire dès lors que c'est une institution de travail de deuil, occupée à collecter de manière un peu nécrologique la mémoire d'un monde qui disparaît... Soit. Mais, en tout cas, notre but est bien de produire de nouvelles sources, des sources permanentes de notre époque. Je ne suis pas sûr qu'un Écomusée - qui fait un travail de représentation de la société industrielle, travail de mémoire pour des gens qui sont encore au contact - soit sur la même problématique quand il contribue à la création d'un centre d'archives. Le plus souvent, il le fait à l'occasion d'une action de sauvetage, et le danger est de n'inscrire cette collecte que dans une action immédiate ou de courte durée, avec le danger de morcellement que j'indiquais tout à l'heure.

\section{Un Centre unique à Roubaix pour une activité sur tout le territoire ?}

15 Question: Il devait y avoir 5 Centres répartis sur le territoire national, il n'y en aura, selon toute vraisemblance, qu'un. Votre rapport au territoire régional est-il transformé par cette modification du projet global d'Archives spécialisées «monde du travail»?

16 La vocation est nationale, la Direction des Archives nous ayant confié un rôle de collecte, de traitement et de communication des archives, mais aussi un rôle de coordination nationale sur ce type d'archives. Dans un nouveau contexte où, faute d'un maillage du 
territoire, nous nous retrouvons seul centre spécialisé, comment concevons-nous notre travail ? Effectivement, alors que nous nous étions assignés, au moment du démarrage, de la préfiguration du Centre, un travail sur la région Nord-Pas-de-Calais, la Picardie, la Champagne, nous collectons maintenant des fonds d'archives d'autres régions. On est amenés à collecter les archives de la société Bull - toute l'histoire de l'informatique depuis l'entre-deux-guerres - on a travaillé avec la Compagnie générale maritime pour trouver des solutions pour ses fonds du Havre; on travaille aussi avec la Compagnie d'entreprises métallurgiques, qui est «la » grosse entreprise de construction d'ouvrages d'arts, en France, mais aussi à l'étranger. Mais cela ne revient pas à tout rapatrier à Roubaix, y installer un monopole de l'archivage économique : ce serait ridicule. Dès lors, notre rôle consiste d'abord à recueillir les fonds d'entreprise que les Archives nationales délocalisent pour donner à Roubaix une dimension suffisante pour être un centre ouvert à la recherche avec une taille critique suffisante. Nous collectons aussi les grandes entreprises à vocation nationale. En revanche, quand on est confrontés à une entreprise d'une autre région, localisée dans sa production, et qui nous contacte pour essayer d'avoir un plan de travail pour sauvegarder ses archives, notre rôle national est d'essayer de trouver des solutions avec les partenaires locaux - archives départementales, municipales -, travailler en coopération avec d'autres institutions non archivistiques aussi, de type associatif, d'économie mixte... On joue alors le rôle de structure de coordination, ayant son expérience comme structure spécialisée.

17 Le rôle national, c'est aussi d'être un centre de ressources méthodologiques. C'est là quelque chose de très important, d'être un lieu où se crée une méthodologie du traitement de ces archives. La « formation » est un volet de notre activité directement lié à ce rôle.

\section{Collecter la mémoire pertinente d'archives privées et y donner accès}

Question: Vous collectez et communiquez des archives issues des acteurs des rapports sociaux de production, quelles spécificités cela induit-il dans votre manière de travailler?

Qu'elles soient d'entreprise ou associatives, ce sont à plus de $90 \%$ des archives privées - je laisse de côté le problème juridique spécifique des grandes entreprises privées, puis nationalisées, puis privatisées... - Nous sommes donc un centre d'archives public, national, dépendant de l'État, mais qui ne collecte que des archives privées! Cela veut dire d'abord que nous n'avons pas à appliquer une réglementation comme on le ferait dans un cadre d'archives publiques. Ici, il n'y a pas d'obligation faite au producteur d'archives de verser régulièrement au Centre. Donc, nous ne pouvons travailler que de manière contractuelle sur une base conventionnelle, autour de contacts créés grâce aux études que nous pouvons faire sur des gisements d'archives existants, par les demandes des producteurs d'archives qui nous arrivent au fur et à mesure que nous sommes connus, par les contacts que nous avons par le biais d'intermédiaires, avec le rôle important des chercheurs dans ce domaine.

Cette base contractuelle impose des méthodes de travail précises. D'abord expliquer quel est notre rôle aux producteurs d'archives privées. En effet, il y a aussi, sur le marché, des entreprises d'archivage: elles proposent des prestations pour le traitement de l'information vivante dans l'entreprise, des archives intermédiaires pour le temps de vie juridique des documents. Nous, nous proposons des prestations patrimoniales, c'est-àdire la conservation définitive de la mémoire pertinente de l'entreprise. Donc, là, il faut 
de la clarté lors du contact. L'organisation de l'information. on peut avoir notre mot à dire, ce n'est pas un sujet d'ignorance ou de désintérêt pour nous : il est très important de comprendre les structures actuelles de l'entreprise ne serait-ce que pour trouver, par méthode régressive, les structures de la mémoire et de la documentation. Mais, ce qui nous anime et qu'il nous faut partager, c'est un souci de la présentation du patrimoine en tant que mémoire de l'activité. A partir de là, on fait du traitement sur le site ; à partir du moment où les documents n'ont plus d'utilité active, on sélectionne ceux qui paraissent importants en tant que mémoire à pérenniser, on établit la convention avec le producteur d'archives.

21 Celle-ci traitera de la propriété (se dessaisit-il de sa propriété, ou - cas le plus fréquent la conserve-t-il en nous confiant le dépôt ?); mais aussi de la communication. Puisque notre mission est de collecter des archives dans l'intérêt de la recherche historique, on a aussi le souci de les rendre communicables dans les meilleurs conditions possibles, alors qu'une entreprise d'archivage qui a une mission de gestion ne se pose pas du tout ce problème.

On propose au producteur d'archives d'appliquer au fond les règles de la loi sur les archives publiques, et parfois même d'anticiper sur certains délais de communication établis par certaines de ces règles. Dans le monde archivistique, on est confronté en général au problème épineux du délai de 60 ans sur les archives dites sensibles... On a le délai de 30 ans, le plus souvent, et... on essaie de faire comprendre au producteur de ces archives que, s'il a une démarche pour préserver sa mémoire, il faut aussi une démarche pour l'ouvrir à la recherche. Mais on n'a pas une réglementation à appliquer, c'est au cas par cas, chaque convention de dépôt peut comporter une solution différente: tel peut demander l'habilitation préalable du chercheur, vouloir réserver la communication pendant tant d'années pour telle ou telle catégorie de documents, ou souhaiter la fermeture complète sur une période... Mais là, on n'innove pas, c'est le principe appliqué pour les archives industrielles aux Archives nationales. Comme archives publiques, notre particularité, c'est de ne travailler que sur une base conventionnelle.

\section{La construction des fonds d'archives et leurs utilisateurs}

Question: Jusqu'ici les usagers des Archives publiques étaient essentiellement des historiens, spécialistes d'histoire économique et sociale, sans compter les généalogistes, bien sûr. Avez-vous un public différent? L'intérêt de nouveaux types de spécialistes, de chercheurs, pour le «monde du travail » et ses archives a-t-il des conséquences sur votre activité ?

Le CAMT, c'est à la fois un centre d'archives et un centre culturel, avec des espaces d'exposition, des salles de conférences: les deux activités ne sont pas à nos yeux étanches; elles peuvent amener ici des usagers intéressés différemment par la mémoire du monde du travail.

En ce qui concerne le public des chercheurs, la demande en documentation très contemporaine est beaucoup plus forte sur un centre d'archives du travail que sur d'autres archives. Parce que, au fond, il y a des mutations de sociétés importantes sur la période et que le chercheur veut, à bon droit, les traiter à partir de documents produits directement par les acteurs du champ d'activité qu'il s'assigne. La création du CAMT va beaucoup accélérer ce phénomène ; et ce n'est pas simple, notamment en ce qui concerne les problèmes de communicabilité. Dans notre domaine donc, il y a une demande de documentation des années 70 , voire 80 , alors qu'à l'heure actuelle les grands débats des 
historiens du monde ouvrier et de l'histoire politique sont centrés sur la Seconde Guerre Mondiale, et bientôt la Quatrième République. Je pense que nous allons devoir nous poser des problèmes d'ouverture de fonds très récents. Et ça pose des problèmes méthodologiques - savoir traiter en temps réel -et des problèmes juridiques.

Mais l'ouverture à la recherche comporte aussi un autre aspect: alors que les archives sont traditionnellement le domaine de l'historien, histoire politique, sociale, économique..., ici, on s'aperçoit, pas à grande échelle certes, que viennent aussi d'autres chercheurs ; des juristes, par exemple, qui s'intéressent au droit de l'entreprise, mais pas à un droit consistant uniquement en textes réglementaires; viennent aussi des sociologues, des ethnologues (le patrimoine industriel est une source d'interrogations), des spécialistes des sciences humaines, comme vous, des sémiologues qui viennent chercher le support d'études sur le discours, sur l'écrit dans l'entreprise. C'est une ouverture intéressante. On s'aperçoit que notre spécialisation, qui aurait pu donner l'impression que nous nous rétrécissons par rapport à un centre d'archives générales, donne en fait des potentialités de débouchés sur l'ensemble des sciences humaines.

C'est intéressant et stimulant; et en même temps, cela nous amène aussi à réfléchir sur les propres méthodes que nous nous donnons pour la collecte... et surtout pour l'échantillonnage des archives.

En effet, la réalité des archives contemporaines, c'est que la masse est telle que, pratiquement, on est amené à conserver un ratio de $10 \%$ de la production. C'est à peu près comme cela dans tous les pays industriels. Alors, dans les archives publiques, on s'est créé des méthodes d'échantillonnage, en conjuguant l'expérience des archivistes et les contacts avec les universitaires; cela a donné une base de travail. On s'aperçoit que l'ouverture à d'autres types de recherches nous amène à nous interroger sur la pertinence de certaines éliminations que nous faisions. Je pense aux parapheurs, aux séries de télex qui sont la circulation interne de l'entreprise. C'est vrai qu'on avait tendance à considérer que c'est un support fragile, et sans contenu informatif très fort... Il est évident qu'il y a de nouvelles entrées, de nouveaux problèmes, cela nous amène à dire qu'il ne faut pas qu'on se " plante ». Car on travaille quand même pour le long terme. On ne peut travailler efficacement que si on a un lien avec les chercheurs, un lien quasi institutionnel. Cela reste à créer, mais il est nécessaire si nous voulons avoir une réflexion en amont sur les phases de collecte et de traitement ${ }^{1}$.

\section{Au-delà de la mise en perspective, pourquoi un chercheur en communication s'intéresserait-il aux archives d'entreprise?}

Je n'ai pas la prétention de faire le point sur l'apport de la dimension historique dans les sciences de la communication, et plus particulièrement dans les études de communication organisationnelle. Nombre d'ouvrages consacrés aux technologies, à leur implantation dans les entreprises s'ouvrent régulièrement par une introduction historique. Beaucoup de chercheurs estiment que les évolutions des organisations doivent être analysées sur une période plus large que les deux dernières décennies. Commencer à citer des noms serait faire injure aux oubliés. Aussi mon propos est moins de situer l'importance actuelle des recherches en communication ayant un tour historique ${ }^{2}$ que d'indiquer les enjeux et 
les difficultés de recherches qui font appel à des archives d'entreprises et d'organisations. J'interviendrai ici d'abord sur les enjeux.

Si beaucoup d'entre nous sont convaincus que la mise en perspective historique des supposées mutations et des prétendues innovations incite les chercheurs en communication à ne pas se précipiter sur la dernière nouveauté avec la naïve allégresse de la pie voleuse, nous n'avons pas pour autant encore partagé publiquement les discussions nécessaires sur la construction de nos données. Combien d'informations nous viennent de nos stagiaires en entreprises et de leur collectes, sans que nous ayons osé, comme a pu le faire M. Villette $(1994)^{3}$, dire les intérêts et les limites de ce mode de construction des données. De même nous n'avons encore que peu discuté des problèmes que nous rencontrons dans l'accès aux documents et à l'observation directe en entreprise, des problèmes particuliers que nous rencontrons, nous, spécialistes des phénomènes de communication qui ajoutons souvent la circulation d'informations induite par nos interrogations à celle, ordinaire, du lieu d'activité. Or nous avons intérêt à envisager ce travail avec d'autant plus de sérieux que nous rencontrons, tout comme les conservateurs des Archives du monde du travail, des éléments fragmentaires de fonds, préconstitués ${ }^{4}$, et communiqués souvent par des acteurs pour qui la mémoire de l'activité est momentanément stratégique ${ }^{5}$. Nous avons à envisager ce travail avec d'autant plus de sérieux que, comme d'autres chercheurs, nous sommes pris dans des débats publics (sur le sens des mutations, sur la valeur des communications actuelles, sur la pertinence des intentions managériales, sur la consistance de professionnalités, etc.) où il est difficile de ne pas dire son mot, de ne pas faire valoir ses hypothèses, ses orientations dans le débat scientifique, là, maintenant, sans que l'on puisse se donner le temps de l'étude, peut-être souhaitable. Disposons-nous de la masse critique de chercheurs qui permettrait de passer de l'étude monographique aux études plus larges? Disposons-nous de typologies d'entreprises et d'institutions qui nous permettraient de construire la confrontation des données? Sommes-nous assez structurés en communauté scientifique pour que nous puissions avoir à l'ordre du jour des questions construites collectivement et permettant, là encore, la confrontation? Ces questions se Posent avec plus d'acuité dès lors que le chercheur accepte la durée longue du travail de terrain et la durée longue du travail d'archives: investir sur le long terme peut avoir comme corollaire une disparition prolongée de la scène de la communication scientifique !

31 Pourtant, le travail d'archives me semble ouvrir à des résultats spécifiques dont j'aimerais signaler l'intérêt.

Il permet d'abord, par la mise en perspective historique, de ne pas être victime de l'illusion de modernité. La formule est classique et un peu vague, donnons un exemple et reformulons: nombre de pratiques professionnelles, notamment en communication, se donnent à voir et s'affichent comme des nouveautés. Or les archives d'entreprise peuvent nous donner à lire les nombreux modèles dont se nourrit la pratique: un dossier de préparation dune journée portes ouvertes « $75^{\mathrm{e}}$ anniversaire » dans un chantier naval en 1975 donne à lire, dans un compte-rendu de réunion, la demande explicite venant de la direction de retrouver l'organisation des cérémonies $\mathrm{du}$ « $50^{\mathrm{e}}$ anniversaire »; la présence d'une affiche « retrouvée » dans le dossier illustre bien cet aspect des pratiques.

Il permet encore de s'ouvrir à des fonds d'entreprises ordinaires et à ne pas être les thuriféraires des seuls services pilotes et innovateurs. La multiplicité des fonds dont on peut disposer au CAMT permet de développer une étude d'entreprises ordinaires ", de services ordinaires. Ainsi, dans le même fonds ${ }^{6}$, on peut comprendre la manière dont s'est 
mis en place tardivement un journal d'entreprise (1957), développé d'une manière " professionnalisée » seulement dans les années 1975, grâce aux communications (conseil, audit, formation) qu'un salarié du service relations publiques a pu (incité à le faire ?) mettre en œuvre avec l'Union des journaux et journalistes d'entreprise de France. Un enjeu: comprendre l'installation, ordinaire et généralisée, de services, de postes, de compétences, de produits de communication.

En troisième lieu, le recours aux études sur fonds d'archives nous permet de nous interroger sur les pratiques communicationnelles. Une méthodologie est à construire, qui commence par l'analyse des traces des communications, dans leur variété, pour tenter de reconstruire les processus. La démarche d'entretiens avec les acteurs est parfois possible, elle se fait selon un autre rapport aux acteurs que dans l'observation directe ou dans la collecte d'informations par les stagiaires. Avec les archives déposées dans un centre d'archives publiques, nous disposons de fonds qui ont échappé au contrôle direct du donateur, de la source; on peut interroger l'acteur en lui donnant une autre place, celle d'expert aidant à la reconstruction d'un objet scientifique dont le point de-départ est le document, la trace et non le souvenir et la défense des intentions.

Enfin, les archives nous permettent d'évoluer dans nos questions de recherche: des corpus peuvent enfin être construits qui donnent la possibilité de poser des questions dont nous devions écarter nos étudiants, faute de faisabilité. De nouveaux types de documents sont accessibles, corpus de photos d'entreprise, ou encore ces séries étonnantes signalées dans le dépliant de présentation du CAMT.

«L'imprimerie Dehon à Valenciennes. Un ensemble exceptionnel de plusieurs dizaines de milliers d'étiquettes et d'emballages de produits alimentaires ou ménagers, des années 1920 à 1992, mis à la disposition des chercheurs et des curieux au fur et à mesure du classement. » travail permet de se doter d'un cadre d'analyse plus riche que celui dont nous disposerions en entrant dans l'entreprise. Pour prendre un exemple, l'analyse des rapports des directions d'entreprise à la "presse d'entreprise " peut être réétudiée de manière nouvelle dès lors que l'on peut, à côté de la classique étude de la parole managériale dans la presse interne ${ }^{7}$, analyser les interventions directoriales au comité d'entreprise, accessible par les comptes-rendus du CE... mais encore ses interventions dans la presse locale, la presse économique nationale, etc.

\section{Le conservateur et ses fonds}

Je reprends à présent un entretien mené avec A. Le Goff, conservateur du patrimoine. Dans ses propos courent trois préoccupations qui permettent de mieux se représenter ce 
que sont les fonds réunis au CAMT et de mieux percevoir l'intérêt de l'échange avec le conservateur. volumineuses de documents ou des épaves que nous devons expertiser dans des conditions matérielles souvent difficiles (caves, greniers, etc.). Il faut encore avoir une idée des procédures et des instruments intellectuels que nous avons à notre disposition pour définir des modalités de tri et d'élimination. En effet, la première chose, c'est de maitriser et d'organiser cette masse d'archives; il est pour nous hors de question d'investir des moyens financiers et humains importants pour collecter des masses volumineuses mais pauvres en informations ou des fonds trop fragmentaires pour être utilisables.

«Il nous faut donc mettre au point une méthodologie de traitement et concevoir des inventaires qui offrent des points d'ancrage pour le chercheur. Une étude sur la typologie des documents nous a paru prioritaire pour préparer les entrées, définir les méthodes de travail et produire des instruments de recherche utilisables par tout public. »

\section{Questions de typologie}

Chaque fonds d'entreprise est une réalité unique. Notre Première démarche, avant d'entreprendre la collecte et le classement est donc de tenter de répondre à quelques questions claires et précises : quelle est la forme juridique de l'entreprise concernée ? Quel est son secteur d'activité? Quelle est sa finalité ? (Est-ce la production de biens destinés à la vente ou le négoce de ces biens ? Est-ce la fourniture de services ?) Quelle est la spécificité de ses activités? Quel est son type de fabrication (non standardisé, par fabrication spécifiée lors du devis, ou standardisé, par fabrication sur catalogue ?) Quel est son rythme de fabrication? Que savons-nous en outre de sa dimension, de son implantation et de sa concentration géographique, de son marché, de son degré de concentration ou d'intégration?

Les documents reçus et produits par l'entreprise dépendent en effet très largement et très concrètement de toutes ces caractéristiques. Ces informations recueillies au préalable facilitent le travail d'appréhension des masses et de compréhension des documents.

Communication et organisation, $10 \mid 2012$ 

documents qui ne relèvent pas d'une obligation juridique. Il existe des «archives stratégiques », des " archives techniques ». Une autre perspective typologique est alors nécessaire. Tous les types de documents, obligatoires ou pas, quel que soit leur support, résultent d'une activité ; il est donc indispensable pour les comprendre d'avoir quelques notions sur l'organisation de l'entreprise. La définition des différentes fonctions de l'entreprise permet alors d'élaborer une méthode de travail pour faciliter la compréhension des fonds. La fonction constitue le pont entre le projet de l'entreprise, c'est-à-dire sa politique et ses objectifs, et les moyens qui lui sont nécessaires pour réaliser ce projet.

Une première description des entreprises par le biais de leurs fonctions a été réalisée par H. Fayol (Administration industrielle et générale, 1916), avec une définition de l'entreprise qui se résume en cinq impératifs (»Prévoir, organiser, commander, coordonner et contrôler »). Cette définition ne prévoit pas de fonction " personnel » ou «gestion des ressources humaines ». Actuellement nous suivons le modèle proposé par Jean Gerbier (Organisation et fonctionnement de l'entreprise, 1983, Lavoisier); nous 
distinguons la fonction vente, la fonction technique et de production, la fonction approvisionnement, la fonction personnel, les fonctions administratives, la fonction finance et comptabilité, enfin, la fonction direction. La fonction est conçue comme une réalité abstraite, non directement observable; elle se distingue de "l'organe", directement observable. Cette analyse des documents sous cet éclairage nous permet de résoudre les énigmes de certains organigrammes. Ainsi les " secrétaires généraux », d'une entreprise à une autre, n'ont pas le même rôle. Dans certaines entreprises, il est important d'identifier l'éminence grise, le bras droit du directeur ou la personnalité forte de la famille. Dans telle grande entreprise textile, toutes les notes passaient par " M. Marcel ». Il contrôlait tout ; même dans les dossiers techniques, on reconnaît sa griffe. On reconnaît aussi ce rôle directorial quand c'est son bras droit qui signe.

\section{Un observatoire des rapports sociaux}

On peut voir aussi, à la lecture de ces archives, l'évolution des mentalités dans les rapports sociaux, notamment avec le personnel. Dans le textile, la construction mécanique, quand on compare sur une longue période, on peut voir le vocabulaire changer en même temps que la façon de considérer le personnel. Dans les années 1920, c'est le comptable qui tient à jour le registre du personnel, et puis, petit à petit, ça va devenir le service des relations industrielles, des relations sociales, des relations humaines, et maintenant des ressources humaines. C'est tout un vocabulaire qui change, mais aussi les objectifs: on peut voir émerger les documents de formation, le journal d'entreprise, les livrets d'accueil, et l'ancêtre des cercles de qualité, ces commissions qui visaient dans les années 1955 à améliorer le rendement. La période d'après guerre, avec l'établissement des comités d'entreprise, voit se développer le souci de la formation professionnelle, de la sécurité, avec la médecine du travail...

On peut voir aussi, à la lecture des archives, l'autre face des rapports sociaux, la méfiance, notamment dans la période des grandes grèves : les services «personnel », ou « relations humaines » collectent tous les tracts, même ceux distribués à la porte des usines, avec des annotations portées sur les conditions dans lesquelles ils ont été distribués. On voit aussi les rapports sur tout ce qui se faisait, les élections...

\section{Des archives de la production propre de documents, mais aussi de «l'information recueillie »}

Il y a en effet un aspect des archives qui est le résultat de l'information qui circule dans l'entreprise. On peut ainsi repérer "l'information de source intérieure", qui va se traduire par des circulaires, des notes de service, des comptes-rendus de réunion. Et il y a "l'information de source extérieure" venant d'autres organismes professionnels, des syndicats patronaux, de l'État. Des coupures de presse sont recueillies, par le service de relations publiques ou par le secrétariat général. Ici encore, on note une évolution, surtout dans les années 1970 : un souci de plus en plus grand de l'image de l'entreprise. Comment l'entreprise est-elle perçue? La question ne se règle plus seulement par les cercles de qualité, à l'interne, par une attention aux rapports sociaux dans l'entreprise. On cherche à savoir, et on collecte pour cela des informations, comment l'entreprise est perçue à l'extérieur. Communiqués de presse et, dans les grandes entreprises, actions de 
mécénat. On trouve ici des informations sur de nombreux supports, y compris les cassettes vidéo où sont enregistrés les grands moments de la vie de l'entreprise, vus par le regard des médias ou par ses propres productions.

\title{
Les relations avec la clientèle
}

Un autre aspect est suggestif et intéressant: les relations avec la clientèle. Ainsi nous avons un fonds d'imprimerie qui comporte la correspondance avec la clientèle sur une très longue période. Le cas est intéressant car, dans cette entreprise, tout était classé par ordre alphabétique de client, dans des casiers, et à la fin de l'année, c'était relié: on gardait tout, aussi bien les bordereaux d'envoi... C'est une source très riche de pratiques de 1945 à 1990. On peut y voir l'évolution géographique de la clientèle, mais on peut aussi bien étudier l'évolution des pratiques d'écriture et de leurs aspects diplomatiques. Un aspect sociologique peut être aussi envisagé : les fichiers clients peuvent avoir un aspect inquisiteur, il s'agit de savoir si les clients sont solvables...

\section{Une sémiologie des écrits d'entreprise}

"L'imprimerie est aussi stratégique pour repérer l'évolution même des produits imprimés des entreprises. On peut lire l'évolution des demandes, l'évolution des techniques de production.

\begin{abstract}
« Dans cette imprimerie qui avait une très large clientèle sur la France, on faisait aussi bien avant guerre de la typographie d'entreprise, imprimant des bulletins de paie, des notices d'information... une très belle collection d'imprimés d'entreprise. Mais, en outre, sa grande spécialité, c'était les impressions couleur : étiquettes pour emballage et cartonnage. On a pu ainsi retrouver des premières étiquettes de clients fort connus, des dossiers de fabrication, de 1923 à 1990. Des dossiers administratifs suivaient le produit de la commande jusqu'à l'envoi, avec des papiers sur la qualité d'encre pour réaliser telle étiquette, etc. Ce qui est intéressant, c'est qu'on a pu conserver les épreuves et un exemplaire de chaque produit fini. On est en train de faire un fichier par client et par produit, et il y en a des milliers et des milliers. Nous avons aussi la correspondance, avec les demandes des clients, en particulier des cas où le travail demandé fait référence au "modèle » d'une autre marque de renom. Bien sûr un tel gisement peut intéresser des maquettistes, mais aussi des sémio-logues, des chercheurs en communication. »
\end{abstract}

57 A. Le Goff, conservateur du patrimoine, Archives du monde du travail. (Entretien réalisé par P. Delcambre, Gérico/Lille 3, février 1995)

Dans une scénographie de l'échange, au risque d'imposer plus qu'il ne faudrait mon expérience comme base de réflexion, j'aimerais signaler ici quelques difficultés que quelques étudiants et moi-même avons rencontré dans un tel travail sur fonds d'archives.

Il faut commencer par dire l'extraordinaire complexité, pour un non-spécialiste de telle ou telle activité, de la compréhension d'un monde professionnel. Les fonds sont bruts, et la compréhension d'un monde industriel, d'une activité sociale commence par la construction du terrain, que l'on entre dans ce monde par les archives ou par l'observation directe. Or la construction du terrain est aussi difficile pour nous que pour nos stagiaires : n'avais-je pas cru, en m'intéressant aux « Chantiers navals de Dunkerque » avoir accès au monde de la marine marchande... La construction de la référence, l'élaboration de documents secondaires pour partager une connaissance minimale 
suffisante d'une activité avec d'autres chercheurs est un travail difficile et long: les sources mêmes n'existent pas forcément pour réaliser ce travail préliminaire. Vouloir comprendre « la communication » renforce cette difficulté : il y a chez nous une ambition de reconstruire les univers des relations, leur économie symbolique... L'ambition est belle-Une première difficulté vient précisément du fait que les $10 \%$ d'archives sauvegardées l'ont été dans le cadre d'une préconstruction d'objet scientifique. Nombre de documents ont disparu, aux Archives comme dans les archives d'entreprise pourraiton dire. Dès lors nous pouvons avoir des difficultés pour reconstruire des pratiques communicationnelles dès lors que la mémoire de certaines communications a été protégée plus que celle d'autres. Ainsi, dans le fonds archivistique du service « relations publiques » des Chantiers de Dunkerque avons-nous des masses de dossiers concernant une des activités du service : l'organisation des lancements « livraisons » et "baptêmes » de navires. Nous n'avons plus trace en revanche des diapos et du texte réalisé pour présenter le Chantier aux visiteurs, pas plus que du canevas de présentation utilisé lors des visites ou encore lors des rencontres avec les classes de collège lorsqu'il s'agissait de les inciter à entrer dans les écoles professionnelles des Chantiers de France. Une attention spécifique aux documents peut certes nous permettre de trouver l'information sensible, telle cette correspondance momentanée entre le responsable du journal et l'UJJEF, ou le programme d'une journée de formation de l'UJTEF à Paris, sur les nouveaux procédés de maquette de journal...

60 Autre difficulté : à partir de ces documents, pour peu que nous cherchions à reconstruire l'activité - non à faire une généalogie des produits de communication - il est difficile de reconstruire ce qu'il en était des missions explicites, du sens que les directions souhaitaient donner à l'activité et ce qu'il en était des marges de manœuvre et des innovations des acteurs. Si une attention à certains types - rares - de documents (lettres de missions, courriers de reproche aux responsables, cadrage de l'activité dans des comptes-rendus de réunions) peut nous aider à reconstruire une partie des enjeux et des débats sur les enjeux des pratiques, il nous faut en fait nous appuyer, quand cela est encore possible, sur une méthodologie associant lecture de documents et interview d'acteurs : des interviews commentant certains documents permettent, avec les limites connues des rationalisations a posteriori, d'avancer dans cette direction.

61 Enfin on signalera une troisième difficulté : les archives, pourtant réduites à $10 \%$ de leur masse, sont encore une masse. Quels sondages mener pour prendre la mesure de l'existant, quels documents définir, a priori et par hypothèse, comme caractéristiques, typiques, stratégiques dans une recherche? Dès lors que l'on ne part pas avec une prédéfinition d'un type de document, mais que l'on cherche à saisir un état des relations et des formes de communication, cette définition des documents susceptibles de nous intéresser est fondamentale : elle est nécessaire dans notre dialogue avec l'archiviste, le bordereau descriptif $\mathrm{du}$ fonds; elle est au principe de notre évaluation des premières recherches et de leur succès. Ainsi, parmi les soixante-dix-sept dossiers d'activité du service relations publiques des Chantiers concernant les lancements de navire, de 1965 à 1987, lesquels étudier? Les études sur corpus propres à la communication organisationnelle, quand elles empruntent les fonds d'archives publiques, nous mettent en face de fonds énormes, à des types de documents d'une extrême variété : des corpus moins maniables que ceux de nos collègues spécialistes de la communication politique et des médias. Un senior finit par «savoir ", mais comment guider l'intuition du jeune chercheur, quels principes donner lors des premiers temps de formation? 


\section{Quelques informations sur les fonds disponibles.} notamment l'état des fonds disponibles, inclut la liste des inventaires édités (en cours d'exploitation, avec possibilité d'exploitation immédiate), l'état des fonds AQ « archives industrielles " provenant des Archives de France, un état des archives privées indiquant pour chaque fonds la nature des documents, les dates extrêmes du fonds et le volume en mètres linéaires. Parmi les fonds accueillis tout récemment, nous en signalerons deux. Je reproduis ici, en accord avec la direction du Centre des archives du monde du travail, deux « dossiers de presse » réalisés à l'occasion de la constitution de ces fonds. Ces deux dossiers de presse peuvent être lus aussi bien comme des exemples du discours construit par les archivistes pour les chercheurs que comme une proposition de travail, informant «à toutes fins utiles» les chercheurs dans le cadre d'une règle de non-concurrence sauvage : les fonds sont accessibles sur la base de déclarations de sujets de maitrise, de doctorat, de thèse.

\section{Le dépôt des archives de la Mission de France à Roubaix}

$\mathrm{M}^{\mathrm{gr}}$ Georges Gilson, archevêque de Sens-Auxerre, prélat de la Mission de France et président de l'Association diocésaine de la Mission de France, et M. Alain ErlandeBrandenburg, directeur des Archives de France, ont apposé leurs signatures le 26 novembre dernier au bas d'une convention de dépôt des archives de la Mission de France au Centre des archives du monde du travail à Roubaix. Conformément à cette convention, la consultation de ces archives, soit environ 400 boîtes de documents témoignant des activités et réflexions des membres de la Mission de France de 1943 à 1985, est soumise à l'autorisation du déposant.

Le choix de Roubaix pour conserver leur mémoire a été expressément voulu par les prêtres de la Mission de France: il s'explique par la vocation nationale du Centre des archives du monde du travail alors que la Mission de France a des points d'ancrage dans près de 50 diocèses de France et une action internationale. Mais il révèle surtout leur engagement, leur volonté "d'être avec le monde du travail" selon leur expression. C'est en effet dans le monde ouvrier et dans les milieux défavorisés qu'ils vivent et travaillent en communauté depuis 1943. C'est dans cet esprit qu'a été créé, en 1941 à Lisieux, leur séminaire sous l'impulsion du cardinal Suhard.

Grâce au bordereau détaillé rédigé par deux des leurs, Paul Collet et Bernard Boudouesque, ce fonds, essentiel pour la connaissance de l'histoire sociale contemporaine, peut déjà être mis à disposition de la recherche historique. D occupe donc maintenant une place de première importance parmi un riche ensemble de fonds collectés par le CAMT depuis 1993, qui témoignent de l'action de l'Église de France dans le monde ouvrier: archives de la CFTC - Nord, de militants jocistes, de militants des mouvements familiaux, de prêtres, ouvriers etc.

\section{Le dépôt des archives du groupe du crédit coopératif à Roubaix}

Les archives historiques du Groupe du Crédit coopératif ont été confiése en dépôt en décembre dernier au Centre des archives du monde du travail, à Roubaix. 
67 C'est un décret-loi du 17 juin 1938 qui a créé la Caisse centrale de crédit coopératif pour reprendre la gestion d'aides accordées auparavant par l'État aux coopératives ouvrières de production et aux coopératives de consommation. Au fil du temps et de diverses réformes, la Caisse centrale de crédit coopératif est devenue l'organe central d'un groupe d'établissements coopératifs de crédit compétent pour l'ensemble de l'Économie sociale : coopératives de toutes natures, mutuelles, associations. Son principal affilié, la Banque française de crédit coopératif, a repris en 1969 les activités de la Banque coopérative des sociétés ouvrières de production fondée en 1893.

En 1983, le Crédit coopératif a créé la «Fondation du crédit coopératif», association loi 1901 devenue « Fondation d'entreprise » en 1992, conformément à la nouvelle législation. Parmi de nombreuses autres opérations dont la réalisation relève du mécénat ou de l'humanitaire, la Fondation soutient et accompagne plusieurs projets en faveur de la recherche en Économie sociale et de sa promotion. Sous l'impulsion de son président M. Jacques Moreau, elle a donc logiquement suscité le dépôt des archives historiques du Crédit coopératif aux Archives nationales.

Les archives historiques du Crédit coopératif comprennent en particulier une large documentation sur les clients et sociétaires du Groupe: de nombreuses coopératives, mutuelles, associations et communautés de travail. À Roubaix, elles seront classées avant l'été 1997 et rendues accessibles grâce à un instrument de travail qui sera publié avec le concours de la Fondation avant le $60^{*}$ anniversaire du Crédit coopératif, qui se tiendra en 1998. Elles permettront ainsi aux chercheurs intéressés par les différentes formes d'expérimentation sociale, comme par l'Économie sociale dans son ensemble, de progresser dans leur connaissance. C'est pourquoi la Fondation a décidé de créer, sans attendre, un prix destiné à la publication d'une thèse de doctorat ou d'un travail de recherche réalisé à partir de ces matériaux. Ce prix sera décerné pour la première fois en 1998 par un jury présidé par Michel Dreyfus, historien et chercheur au CNRS.

\section{NOTES}

1. Cet entretien a été publié en mai 1995 dans Études de Communication $\mathrm{n}^{\circ} 16$, Pratiques d'écriture et champs professionnels (3), Petites fabriques d'auteurs, université de Lille 3, p. 183-188

2. Une telle étude bibliographique pourrait erre menée par le Groupe de recherche en communication organisationnelle de la SFSIC

3. VilletteM., L'Art du stage en entreprise, Paris, La découverte, 1994.

4. G. Mouradian, comme archiviste, est souvent confronté à des fonds qui se présentent comme "généraux ", une masse de documents de natures diverses, et non comme un quasi- corpus, et pourtant il signale : «il est très important de comprendre les structures actuelles de l'entreprise ne serait-ce que pour trouver, par méthode régressive, les structures de la mémoire et de la documentation. »

5. Parmi les acteurs, les archivistes d'entreprise eux-mêmes (on connaît le remarquable travail des archivistes de St-Gobain, de la CGM, du ministère des Finances, etc.) et les historiens d'entreprise (ainsi ClioMédia.) auxquels les premiers peuvent faire appel pour certaines études. 
6. Les quelques références au fonds des Chantiers navals de Dunkerque ont été longuement exposées dans Un service de communication en entreprise et ses histoires aux Premières journées du Groupe de recherche en communication organisationnelle, Rennes, juin 1996, a paraître aux PUR (Ch. Le Moënne, éd).

7. Sur ce point, une confrontation permettrait a l'auteur de ces lignes d'être sûr qu'il existe désormais une étude «classique» des interventions des directions dans la presse d'entreprise, dans les méthodes, sinon dans les résultats !

\section{RÉSUMÉS}

Depuis 1993 est ouvert à Roubaix (59) le Centre des archives du monde du travail. Un chercheur en sciences de la communication présente ici le travail de la direction de ce centre, en deux entretiens, donne quelques informations utiles et s'interroge sur les enjeux et les difficultés d'un travail d'archives pour les chercheurs en sciences de la communication.

Since 1993, the «Centre des Archives du Monde du Travail » hos opened in Roubaix (59). Through two interviews, a communication science researcher explains how does the office work. He gives useful information and underlines the stakes and difficulties for communication science researchers to work on archives.

\section{AUTEUR}

\section{PIERRE DELCAMBRE}

Professeur en sciences de la communication, responsable du DEA de Lille 3/Valenciennes, Pierre Delcambre est membre du groupe de recherches « communication organisationnelle » de la Société française des sciences de l'information et de la communication. Il développe une analyse des pratiques de communication ordinaires (communications de travail, communications publiques) d'entreprises et d'organisations (Travail social, Chantiers navals, Marine marchande). Il cherche à définir les formes stables et les fonctions des écrits professionnels. 